\title{
Diuretika-Missbrauch bei chronifizierter Bulimia nervosa - Fallbericht und klinisches Management
}

\author{
Diuretic-Abuse in Chronic Bulimia Nervosa - Case Report and Clinical \\ Management
}

Autoren

Institut
Martin Greetfeld, Nicole Bröckel-Ristevski, Markus Fumi, Ulrich Cuntz, Ulrich Voderholzer

Schön Klinik Roseneck, Psychosomatik, Prien am Chiemsee
Schlüsselwörter

- Bulimia nervosa

- Diuretika

- Missbrauch

Keywords

- bulimia nervosa

- diuretics

- abuse eingereicht 23. Januar 2015

akzeptiert 20. Februar 2015

Bibliografie

DOI http://dx.doi.org/

10.1055/s-0035-1548780

Online-Publikation: 3.6.2015

Psychother Psych Med 2015;

65: 379-382

(c) Georg Thieme Verlag KG

Stuttgart · New York

ISSN 0937-2032

Korrespondenzadresse

Dr. Martin Greetfeld

Schön Klinik Roseneck

Psychosomatik

Am Roseneck 6

83209 Prien am Chiemsee

mgreetfeld@schoen-kliniken.de

\section{Zusammenfassung \\ $\nabla$}

Wir berichten den Fall einer im medizinischen Bereich tätigen Patientin mit langjährigem Furosemid-Missbrauch im Rahmen einer chronischen Bulimia nervosa (BN). Die Häufigkeit eines Purging-Verhaltens durch die Einnahme von Diuretika bei BN ist aufgrund einer hohen Verheimlichungstendenz der Betroffenen schwer zu erfassen; bei 10\% scheint es zu einem längerfristigen schädlichem Gebrauch zu kommen. Bedingt durch die pathophysiologischen Veränderungen mit Hyperaldosteronismus kommt es beim Absetzen der Diuretika zu einer Ödementwicklung, die - sei es bedingt durch die Gewichtsphobie oder intolerable Spannungsgefühle v.a. im Gesicht oder in den Beinen - zu einer erneuten Eskalation des Purgingverhaltens führen kann. Die gute Kenntnis der pathopysiologischen Zusammenhänge stellt hier Basis für ein adäquates klinisches Management dar, das aus Psychoedukation und Informationsvermittlung, Behandlung der Elektrolytstörungen und in Einzelfällen der Gabe von Aldosteronantagonisten besteht.

\section{Einleitung}

\section{$\nabla$}

Kernsymptome der Bulimia nervosa (BN), die mit einem Verhältnis von 20:1 überwiegend Frauen betrifft und für die eine Lebenszeitprävalenz bei Frauen von ca. 1,5\% angegeben wird, sind wiederholte Essanfälle und gegensteuernde Maßnahmen [1]. Diesem Verhalten liegen eine übertriebene Beschäftigung mit dem Körpergewicht und ein ausgeprägtes Schlankheitsstreben zugrunde. Bei den gegensteuernden Maßnahmen handelt es sich meistens um selbst induziertes Erbrechen, aber auch um die missbräuchliche Einnahme von Laxantien oder Diuretika (ICD-10 [2]). Sowohl Medikamente im engeren Sinn, als auch Tees, Nahrungsergänzungsmittel und andere,

\section{Abstract \\ $\nabla$}

We give account of a patient, who works in health care, with bulimia nervosa (BN) and a long term abuse of Furosemide. Due to patients' tendency to conceal addictive behavior and symptoms of BN, the prevalence of purging behavior caused by the intake of diuretics is difficult to quantify $10 \%$ of BN patients exhibit a long-term harmful abuse. Discontinuation of diuretics causes the development of edema, attributable to pathophysiological changes with hyperaldosteronism. These can lead to renewed escalation of purging behaviour, provoked either by phobia of weight gain or by unbearable feelings of tension in the facial area or in the legs. For an adequate clinical management, it is vital to have thorough knowledge of the pathophysiological context which consists of psychoeducation, provision of information, treatment of water-electrolyte imbalance and, in individual cases, the administration of aldosterone antagonists.

auch außerhalb von Apotheken frei verkäufliche Substanzen werden zum Abführen, Entwässern oder für eine vermeintliche Anregung des Grundumsatzes genutzt [3]. Körperliche Folgeerscheinungen des gegensteuernden Verhaltens sind bei Patientinnen mit BN häufig zu beobachten (z.B. Elektrolytverschiebungen und Ödeme als Folge von Salzverlusten oder Zahnschäden und Schwellungen der Parotis als Folge des Erbrechens).

Die ersten Berichte über eine missbräuchliche Einnahme von Diuretika finden sich Anfang der 1970er Jahre in der Literatur. Die primär aus internistischem Blickwinkel verfassten Berichte beschreiben ein durch Diuretika verursachtes Bartter-Syndrom (charakterisiert durch Hypokaliämie, Alkalose, Hyperreninämie, Hyperaldoste- 
ronismus, normalen Blutdruck und Hyperplasie des juxtaglomerulären Apparats der Niere), das in Abgrenzung zu der idiopathischen Variante, bei dem eine kongenitale Schädigung der renalen Ionentransportmechanismen zugrunde liegt, später die Bezeichnung Pseudo-Bartter-Syndrom erhielt [4]. In Einzelfällen wurde der Diuretikamissbrauch mit Magersucht in Verbindung gebracht [5]. Im Rahmen von Veröffentlichungen zu sogenannten „idiopathischen Ödemen“, einem nach Ansicht der damaligen Autoren primär bei Frauen auftretenden Phänomen, wurde ebenfalls Diuretikamissbrauch beschrieben. Weitere Beobachtungen, z.B. dass diese normalgewichtigen Frauen quasi „von der Beschäftigung mit dem Körpergewicht besessen seien“, waren damals noch keiner eigenen diagnostischen Entität im Rahmen psychischer Erkrankungen zuzuordnen (zur Übersicht [6]). Dies änderte sich erst mit der Erstbeschreibung der BN durch Russell 1979 [7].

Jüngere Berichte beschreiben Fälle ungeklärter chronischer Hypokaliämien (teilweise auch erheblicher Ausprägung bis unter $2 \mathrm{mmol} / \mathrm{l}$ ), für die letztendlich ein Diuretika-Missbrauch verantwortlich gemacht werden konnte $[8,9]$. Eine nephrologisch geführte Fallserie beschreibt 18 Fälle von teils extrem langem Furosemid-Missbrauch (bis 25 Jahre); insbesondere hohe Dosen (im Mittel um über $500 \mathrm{mg}$ Furosemid pro Tag) waren mit einer bilateralen medullären Nephrokalzinose vergesellschaftet [10]. Dass in den Fallberichten vor allem ein Missbrauch des Schleifendiuretikums Furosemid, das im aufsteigenden Teil der Henle-Schleife die Natrium-Rückresorption hemmt und somit stark entwässernd wirkt, berichtet wird, liegt am ehesten an der hohen Potenz und den damit verbundenen drastischen Auswirkungen auf den Wasser- und Elektrolythaushalt, der letztendlich zu Notfallaufnahmen in somatische Krankenhäuser führt. Wie häufig Diuretikamissbrauch bei BN ist, ist letztendlich nicht bekannt. Gründe hierfür sind, dass auch trotz gründlicher Anamneseerhebung Patientinnen den Gebrauch von Diuretika nicht angeben oder mildere diuretische Maßnahmen wie die Einnahme von Entwässerungstees nicht entsprechend einordnen. Selbst in der S3-Leitlinie „Diagnostik und Therapie der Essstörungen“ [1] wird keine Häufigkeit des Phänomens angegeben. In einer älteren Studie wurde die Häufigkeit von Diuretika-Einnahme bei College- und High-School Studenten allgemein mit 3-5\% angegeben [11]. Eine US-amerikanische Arbeitsgruppe publizierte zwei Arbeiten mit Angaben zum Diuretikamissbrauch bei Patientinnen mit BN, die sich in medizinischer Behandlung befanden. In der älteren Arbeit $(n=275)$ wird beschreiben, dass ca. 34\% zumindest einmalig diuretische Substanzen (von klassischen Diuretika bis hin zu over-the-counter-Medikamenten, Phytopharmaka und Koffein) zur Gewichtskontrolle eingesetzt hatten und ca. $10 \%$ dies auch über eine längere Zeit taten [12]. In der jüngeren Arbeit $(n=39)$ wird für den gelegentlichen Gebrauch eine Häufigkeit von ca. $30 \%$, für einen Dauergebrauch von ca. 6\% angegeben [3]. Aufgrund der hohen Dunkelziffer bleiben diese Angaben aber allesamt vage.

\section{Kasuistik}

$\nabla$

Wir berichten den Fall einer 42-Jährigen im medizinischen Bereich tätigen Patientin mit langjähriger BN, die Hilfe in unserer auf Essstörungen spezialisierten, verhaltenstherapeutisch orientierten psychosomatischen Klinik suchte.

Die Patientin litt seit über 20 Jahren unter einer Essstörung. Nachdem im jüngeren Erwachsenenalter zunächst anorektische
Verhaltensweisen dominierten (Minimalgewicht bei BMI 16 im Alter von 20 Jahren) kam es im Zuge einer konfliktreichen Ehe vor 10 Jahren zum Auftreten abendlicher Essanfälle mit Erbrechen und einem restriktiven Essverhalten tagsüber bei gleichzeitigem Normalgewicht. Darüber hinaus nahm sie ebenfalls seit gut 10 Jahren zur Gewichtsregulation Furosemid $180 \mathrm{mg}$ pro Tag ein; anfänglich hatten niedrigere Dosen ausgereicht, im Verlauf musste sie die Dosis jedoch aufgrund von Wirkverlust deutlich erhöhen. Komorbid bestand eine ausgeprägte depressive Symptomatik. Bei Aufnahme wog sie $57 \mathrm{~kg}$ bei $1,67 \mathrm{~m}$ Körpergröße (BMI 20,4). Im Eating Disorder Inventory-2 (EDI-2) [13] zeigten sich in allen Skalen auffällige Werte im Vergleich zur Normstichprobe. Der Summenwert des Beck Depressions-Inventars (BDIII) [14] lag bei 39 Punkten, entsprechend einer schweren depressiven Symptomatik. Sie ging von Beginn der Therapie an sehr offen mit dem Missbrauch von Furosemid um und äußerte den klaren Wunsch, die Substanz abzusetzen. Sie berichtete von mehreren gescheiterten Absetzversuchen, wobei jeweils die einsetzende Ödementwicklung für sie nicht tolerabel gewesen sei und zu einer erneuten Furosemid-Einnahme geführt hatte. Die medizinisch versierte Patientin beschrieb klar eine große innere Diskrepanz zwischen dem starken Drang, Diuretika einzunehmen, und dem medizinischen Faktenwissen um die Schädlichkeit dieses Verhaltens. Kurz vor Aufnahme hatte sie durch einen Nephrologen noch eine erweiterte Diagnostik (24h-Creatinin-Clearance, Bestimmung von Renin und Aldosteron) durchführen lassen, jeweils mit unauffälligem Ergebnis.

Im Labor fiel bei Aufnahme eine Hypokaliämie von 3,0 mmol/1 auf, die oral ausgeglichen werden konnte (Kalium $40 \mathrm{mmol} / \mathrm{die}$ in retardierter Galenik über 10 Tage). Der Natrium-Wert war mit $140 \mathrm{mmol} / \mathrm{l}$ normal, Chlorid mit $99 \mathrm{mmol} / \mathrm{l}$ im unteren Referenzbereich. Kreatinin $(0,8 \mathrm{mg} / \mathrm{dl})$ und die 24 -h-Eiweisausscheidung waren normal. Eine Erhöhung der CK fand sich nicht. Sonografisch zeigten sich Hinweise auf eine Nephrokalzinose beidseits. Wir schlugen der Patientin der Empfehlung des internistischen Konsiliars folgend ein abruptes Absetzen von Furosemid und den Ersatz durch Spironolacton vor; diesem Vorgehen konnte die Patientin aber angstbedingt nicht zustimmen, sodass eine langsame, schrittweise Reduktion der Furosemiddosis von $180 \mathrm{mg}$ auf zuletzt $20 \mathrm{mg}$ pro Tag erfolgte, wobei die wöchentlichen Reduktionsschritte zu Beginn (60 mg Reduktion) größer als die letzten Schritte (20 mg Reduktion) waren. Die Befürchtung hinsichtlich des abrupten Absetzens von Furosemid war, ein Nierenversagen zu erleiden. Die Patientin gab an, im Rahmen ihrer eigenen beruflichen Tätigkeit bereits bei ihren Patienten ein Nierenversagen nach Absetzen von Furosemid beobachtet zu haben. Bei unserer Patientin kam es während des Absetzens zu einer Entwicklung milder Ödeme, die keiner weiteren Therapie bedurften. Das Körpergewicht stieg passager maximal um $2 \mathrm{~kg}$. Unter der engmaschigen therapeutischen Betreuung zeigte sich kein restriktives Essverhalten; es gelang der Patientin vielmehr, auch während des Absetzens ausreichende Nahrungsmengen zu sich zu nehmen und nicht zu erbrechen. Zuletzt fanden sich normale Elektrolytwerte bei unverändert normalem Kreatinin. Im Rahmen des störungsspezifischen Behandlungsprogramms gelang es der Patientin, Essanfälle vollständig einzustellen. Wesentliche aufrechterhaltende Faktoren für die langjährige Essstörung waren Einsamkeit sowie konflikthafte, abhängige Partnerbeziehungen und damit zusammenhängende Probleme der Nähe und Distanzregulation, wobei die Symptomatik (bulimisches Essverhalten, Diuretikaeinnahme) als Strategie zum Umgang mit als unangenehm erlebten Gefühlen wie Angst, Wut und 
Einsamkeit diente. Die Diuretikaeinnahme erfolgte auch, um einer Ödementwicklung entgegenzuwirken. Die Ödementwicklung war für die Patientin besonders aversiv, da durch Schwellungen insbesondere im Gesicht und an den Unterschenkeln das Gefühl, zu dick zu sein, verstärkt wurde. Diese Zusammenhänge konnte die Patientin gut reflektieren und profitierte sehr vom Aufbau alternativer Denk- und Verhaltensmuster (z. B. Erarbeitung eines Teufelskreismodells, Aufbau eines geregelten Essveraltens mit ausreichenden Portionsgrößen unabhängig von der aktuellen Wahrnehmung des Körpers).

Die noch bestehende Dosis von $20 \mathrm{mg}$ Furosemid wollte die Patientin über einen längeren Zeitraum zusammen mit ihrem behandelnden Nephrologen am Heimatort reduzieren, da ein längerer Aufenthalt in unserer Klinik der Patientin aus beruflichen Gründen nicht möglich war. Aus nephrologischer Sicht wäre ein rasches Absetzen möglich gewesen. Da jedoch keine unmittelbare Notwendigkeit vorlag, folgte der Nephrologe am Heimatort dem Wunsch der Patienten nach einer gestuften Reduktion von Furosemid. Der BMI bei Entlassung betrug $20,2 \mathrm{~kg} / \mathrm{m}^{2}$.

\section{Diskussion}

$\nabla$

Wir berichten von einem Fall einer im medizinischen Bereich tätigen Patientin mit einer chronischen BN und einem Missbrauch von Diuretika. Bei strenger Interpretation der ICD-10-Kriterien der Abhängigkeit finden sich auch Charakteristika eines Abhängigkeitssyndroms wie psychische Entzugserscheinungen (Ängste und Unruhe), eine Dosissteigerung über viele Jahre mit Toleranzentwicklung, misslungene Versuche des Absetzens und - wenn man die Ödementwicklung entsprechend wertet - auch körperliche Entzugsphänomene. Ein Lustempfinden bei der Einnahme wie bei anderen stoffgebundenen Süchten lag jedoch nie vor. Formal wird in der ICD-10 der Diuretikamissbrauch unter F55.8 „schädlicher Gebrauch von sonstigen nichtabhängigkeitserzeugenden Substanzen“ kodiert [2].

Wie auch in vorliegendem Fall findet sich das Purging-Verhalten über Diuretika häufig bei Frauen mit Essstörungen aus medizinischen Berufen [8]. Wie bereits ausgeführt, wird meist eine Einnahme von Furosemid berichtet. Unsere Patientin nahm mit $180 \mathrm{mg}$ pro Tag eine - im Vergleich zu anderen Berichten über Diuretikamissbrauch - mittlere Dosis ein. Günstig war die hohe Motivation und Compliance der Patientin, sodass eine in anderen Fällen berichtete Bagatellisierung und Leugnung kein Problem darstellte. Erschwerend war jedoch die starke Angst vor dem vollständigen Absetzen von Furosemid; als medizinisch Gebildete sorgte sich die Patientin vor einer vollständigen Dekompensation ihrer Nierenfunktion, da sie entsprechende Erfahrungen bei intensivmedizinisch behandelten Patienten gemacht hatte. Somit konnte das Therapieziel des vollständigen Absetzens im stationären Rahmen nicht erreicht werden.

Pathophysiologisch entsteht bei chronischem Diuretikagebrauch (und anderem intensiven Purgingverhalten) ein Pseudo-Bartter-Syndrom, definiert als ein exogen verursachter Zustand mit Hypokaliämie, Hypochloridämie, Alkalose, Hyperaldosteronämie und Hyperplasie des juxtaglomerulären Apparats der Niere. In Abgrenzung zur idiopathischen Variante fehlt die intrinsische Pathologie (defekte Chloridkanäle) und der Chloridgehalt im Urin ist normal. Die Aldosteronwirkung am distalen Tubulus und am Sammelrohr führt zu einer Reabsorbtion von Natrium im Austausch für Kalium (Folge Hypokaliämie) und Protonen
(Folge Alkalose) (zur Übersicht [15]). Auch nach Absetzen der Diuretika (oder anderen Purging-Verhaltens) bleibt die Überaktivität des Renin-Angiotensin-Aldosteron-Systems bis zu zwei Wochen bestehen, sodass Ödeme und Hypokaliämieneigung resultieren. Die Ödembildung (bis zu $10 \mathrm{~kg}$ in wenigen Tagen) beunruhigt sowohl Patientinnen als Therapeuten häufig sehr stark und stellt einen Risikofaktor für einen Rückfall hinsichtlich des Purgingverhaltens dar. In der S3-Leitlinie wird in milderen Fällen eine abwartende Haltung mit Aufklärung der Patientin über die Natur des Pseudo-Bartter-Syndroms empfohlen. Auch im vorliegenden Fall kam es nur zu einer leichten Ödementwicklung, gegebenenfalls im Zusammenhang mit der langsamen Furosemid-Dosisreduktion. Bei hohem Leidensdruck kann punktuell - entsprechend der beschriebenen Pathophysiologie - der Aldosteronantagonist Spironolacton gegeben werden. Allerdings trägt die Spironolactongabe letztendlich nur zur Verzögerung der Anpassungsprozesse in der Niere bei [1].

Psychotherapeutisch stehen zum einen psychoedukative Elemente in der Akutbehandlung des Diuretikamissbrauchs im Vordergrund, zum anderen die notwendige Verhaltensänderung mit Absetzen der Diuretika, im Zuge derer aufkommende unangenehme Gefühle toleriert werden sollen. Ziel ist, pathophysiologische Zusammenhänge auf verständliche Weise der Patientin näher zu bringen, die Toleranz für die auftretenden Ödeme zu erhöhen, Abstinenz zu erzielen und die Rückfallgefahr zu vermindern. Langfristig sollte auch das Thema der Beschaffung von Diuretika angesprochen werden: Gerstein und Woodside beschreiben beispielsweise ein Behandlungsprotokoll, das auch die Information der verschreibenden Ärzte über den Missbrauch durch die Patientin selbst (z. B. in Form eines Briefs, in dem darum gebeten wird, künftig keine Verschreibungen mehr durchzuführen) vorgesehen ist [16]. Bei medizinischem Personal bleibt die Sicherstellung einer langfristigen Abstinenz aufgrund der leichten Zugänglichkeit der Substanzen eine erhebliche Herausforderung an die psychotherapeutische Arbeit.

In jedem Fall sollten Patientinnen mit BN - insbesondere bei auffälligen Laborkonstellationen - hinsichtlich einer Einnahme von Diuretika oder anderer entwässernder Stoffe gezielt exploriert werden. Der offene Umgang mit dem Thema erhöht die Chance, auch in der problematischen Phase der Ödementwicklung bei Reduktion des Purgingverhaltens die Compliance zu sichern und somit einen guten Behandlungserfolg zu erzielen.

\section{Fazit für die Praxis}

Ca. $10 \%$ der Patientinnen mit Bulimia nervosa betreiben einen chronischen Diuretika-Missbrauch. Aufgrund der hohen Verheimlichungstendenz ist eine gezielte Exploration dieser Art des Purging-Verhaltens nötig. Die Ödementwicklung bei Absetzen der Diuretika wird von den Patientinnen häufig als stark belastend erlebt. In dieser Therapiephase ist eine intensive psychotherapeutische und internistische Betreuung mit Informationsvermittlung über die pathophysiologischen Zusammenhänge im Hinblick auf die vorübergehende Natur der Ödementwicklung, die Behandlung etwaiger Elektrolytstörungen und in Einzelfällen auch die Gabe von Aldosteronantagonisten angezeigt.

Interessenkonflikt: Die Autoren geben an, dass kein Interessenkonflikt besteht. 


\section{Literatur}

1 Arbeitsgemeinschaft der wissenschaftlichen medizinischen Fachgesellschaften (AWMF). S3-Leitlinie Diagnostik und Therapie der Essstörungen (12.12.2010). Im Internet: http://www.awmf.org/leitlinien/ detail/11/051-026.html Stand: 7.3.2015

2 Dilling $H$, Mombour W, Schmidt $M H$. Internationale Klassifikation psychischer Störungen: ICD-10; Kapitel V (F); Klinisch-diagnostische Leitlinien. Bern, Göttingen, Toronto, Seattle: Huber 2011

3 Roerig JL, Mitchell JE, de Zwaan $M$ et al. The eating disorders medicine cabinet revisited: A clinician's guide to appetite suppressants and diuretics. Int J Eat Disord 2003; 33: 443-457

4 Ben-Ishay $D$, Levy $M$, Birnbaum $D$. Self-induced secondary hyperaldosteronism simulating Bartter's syndrome. Isr J Med Sci 1972; 8: $1835-1839$

5 Davidson C, Silverstone T. Diuretic dependence. Br Med J 1972; 1: 505

6 Mitchell JE, Pomeroy C, Seppala $M$ et al. Pseudo-Bartter's Syndrome, Diuretic Abuse, Idiopathic Edema, and Eating Disorders. Int J Eat Disord 1988; 7: 225-237

7 Russell G. Bulimia nervosa: An ominous variant of anorexia nervosa. Psychol Med 1979; 9: 429-448

8 Ruisz W, Stöllberger C, Finsterer J et al. Furosemide-induced severe hypokalemia with rhabdomyolysis without cardiac arrest. BMC Women's Health 2013; 13: 1-4
9 Brener ZZ, Medvedovsky B, Winchester JF et al. Hypokalaemia in a woman with eating disorder. Grand Rounds 2011; 11: 53-55

$10 \mathrm{Kim}$ YG, Kim B, Kim MK et al. Medullary nephrocalcinosis associated with long-term furosemide abuse in adults. Nephrol Dial Transplant 2001; 16: 2303-2309

11 Halmi KA, Falk JR, Schwartz E. Binge-eating and vomiting: A survey of a college population. Psychol Med 1981; 11: 697-706

12 Mitchell JE, Hatsukami D, Eckert ED et al. Characteristics of 275 patients with bulimia. Am J Psychiatry 1985; 142: 482-485

13 Paul T, Thiel A. Eating Disorder Inventory-2 (EDI-2): Deutsche Version. Göttingen: Hogrefe 2005

14 Beck TA, Steer RA, Brown GK et al. Beck-Depressionsinventar - BDI-II. Göttingen: Hogrefe 2006

15 Mascolo M, Chu ES, Mehler PS. Abuse and clinical value of diuretics in eating disorders therapeutic applications. Int J Eat Disord 2011; 44: $200-220$

16 Gerstein F, Woodside B. Diuretic abuse in eating disorders: Treatment protocol and case report. Eat Disord 1997; 5: 184-190 